\title{
Rectus Sheath Block (RSB) Analgesia Could Enhance Significantly the Patient Satisfaction Following Midline Laparotomy in Benign Disease and in Cancer: A Prospective Study With Special Reference to Nitrosative Stress Marker Nitrotyrosine (NT) Plasma Concentrations
}

\author{
VIIVI KUOSMANEN ${ }^{1 *}$, IINA SAIMANEN $^{1 *}$, DINA RAHKOLA $^{1}$, JARI KÄRKKÄINEN $^{1}$, TUOMAS SELANDER $^{2}$, \\ MARTIN PURDY ${ }^{1}$, HANNU KOKKI $^{3}$, MERJA KOKKI $^{3}$ and MATTI ESKELINEN ${ }^{1}$ \\ ${ }^{1}$ Department of Surgery, Kuopio University Hospital and School of Medicine, \\ University of Eastern Finland, Kuopio, Finland; \\ ${ }^{2}$ Science Service Center, Kuopio University Hospital, Kuopio, Finland; \\ ${ }^{3}$ Department of Anaesthesia and Operative Services, Kuopio University Hospital and School of Medicine, \\ University of Eastern Finland, Kuopio, Finland
}

\begin{abstract}
Background/Aim: Our hypothesis was that rectus sheath block (RSB) analgesia could enhance satisfaction following midline laparotomy in patients with benign disease and cancer patients. Patients and Methods: Initially, 56 patients were randomized into four groups; control group $(n=12)$, single-dose $(n=16)$, repeated-dose $(n=12)$ and continuous infusion $(n=16)$ RSB analgesia groups. The plasma concentrations of the NT marker were measured just before, immediately after and $24 \mathrm{~h}$ after operation. Patient satisfaction at $24 \mathrm{~h}$ postoperatively was filed on a 11-point numeric rating scale $\left(\mathrm{SFS}_{24} ; 0=\right.$ fully unsatisfied; $10=$ fully satisfied). Results: The RSB analgesia significantly enhanced the $\mathrm{SFS}_{24}$ scores in the study groups $(p=0.001)$. The median plasma NT concentrations ( $\mathrm{pg} / \mathrm{ml}$ ) following surgery (POP 1$)$ were significantly lower in patients with cancer versus patients with benign disease (5.3 vs. 7.6, p=0.008). Jitter plots of the individual $\mathrm{SFS}_{24}$ values versus plasma NT concentrations were significantly correlated in benign and cancer patients ( $r=-0.284, p=0.028)$. Conclusion: The RSB analgesia could significantly enhance patient satisfaction
\end{abstract}

*These Authors contributed equally to this study.

Correspondence to: Matti Eskelinen, MD, Ph.D., School of Medicine, University of Eastern Finland, P.O. Box 100, FI-70029 KYS, Finland. Tel: +358 17173311, Fax: +358 17172611, GSM: +358 400969444, e-mail: matti.eskelinen@kuh.fi

Key Words: Benign disease, cancer, plasma nitrotyrosine, nitrosative stress, satisfaction following surgery. following midline laparotomy. Plasma NT concentrations versus patient satisfaction following surgery are significantly correlated in benign disease and cancer.

Today, the benefits of laparoscopic surgery (LS), among which are less postoperative pain, shorter hospital stay and sick leave duration, are undeniable. However, from the patient's point of view, the symptom resolution, quality of life and patient satisfaction are essential. Although the proportion of LS in general surgery was $47-97 \%$ in a Swedish register study (1), the proportion of LS in major resection surgery was only between 4-10\% including esophago-gastric, liver, colorectal and pancreatic operations (1). Therefore, the midline laparotomy is still needed in cancer and emergency surgery, when the whole peritoneal cavity is necessary to be explored thoroughly. The midline laparotomy patients need proper analgesia peri- and postoperatively and it would be important to predict patients with a higher analgesia requirement.

Our previous article showed that the control group of patients and the rectus sheath block (RSB) groups did not differ in individual pain scores (numeric rating scales, NRS) following surgery (2). Saimanen et al. (3) reported a significant correlation between the plasma oxidative stress biomarker catalase (CAT) levels following surgery and the individual NRS pain scores 24 $\mathrm{h}$ postoperatively (NRS24) in patients with cholelithiasis. However, a nitrosative stress biomarker nitrotyrosine (NT) plasma concentrations in midline laparotomy patients with benign disease versus cancer is unknown. Arsalani-Zadeh et al. (4) and Yiannakopoulou et al. (5) revised the literature to compare Reactive Oxygen Species (ROS)/Reactive Nitrogen Species (RNS) in open surgery (OS) and LS, and they found 
sixteen clinical trials that investigated the impact of OS and LS on ROS/RNS, however, there were no studies available of plasma NT measurement. Therefore, the present study investigated the NT plasma concentrations in patients with benign disease versus cancer following midline laparotomy. The main hypothesis was that RSB analgesia could enhance satisfaction following midline laparotomy in benign and cancer patients. Secondly, our aim was to determine plasma NT levels in patients with benign disease versus patients with cancer and determine the association between plasma NT concentrations and patient satisfaction $24 \mathrm{~h}$ following surgery $\left(\mathrm{SFS}_{24}\right)$.

\section{Patients and Methods}

The study was approved by the Ethics Committee of the Northern Savo Hospital District, Kuopio, Finland (DNRO 120/2011, November 11, 2011) and was registered in the EudraCT database (EudraCT number 2011-005136-25, Consort diagram, Figure 1) and in the ClinicalTrials.gov database (ClinicalTrials.gov Identifier: NCT02869841). It was conducted in accordance with the Declaration of Helsinki. Participants gave written consent after receiving verbal and written information.

Operations were carried out at Kuopio University Hospital, Kuopio, Finland between 2012 and 2015. The CONSORT flowchart of the study is presented in Figure 1. The study design was a prospective, randomised, clinical trial with four parallel groups. The patients with midline laparotomy were randomized into the control group or into one of the three active groups; single-dose, repeateddose or continuous infusion RSB analgesia groups. The study patients had intravenous oxycodone pumps as the patient- controlled analgesia (PCA). The randomisation list was generated by computer (www.randomization.com), a sealed enveloped method was used for blinding and randomisation was done preoperatively.

The primary outcome measures were the plasma concentrations of $\mathrm{Cu} / \mathrm{Zn}$ superoxide dismutase (SOD1), catalase (CAT) and NT markers measured at three time points with high-sensitivity assays: before (PRE), immediately after (POP1) and $24 \mathrm{~h}$ after surgery (POP2) in the control versus the three active groups. The patient satisfaction at $24 \mathrm{~h}$ postoperatively were filed on a 11-point numeric rating scale $\left(\mathrm{SFS}_{24} ; 0=\right.$ fully unsatisfied; $10=$ fully satisfied) and the overall pain at rest $\mathrm{NRS}_{\mathrm{r}}$ and at $2 \mathrm{~kg}$ pressing $\mathrm{NRS}_{\mathrm{p}}$ surveyed and filed on a 11-point numeric rating scale at $24 \mathrm{~h}$ following surgery (NRS; $0=$ no pain; $10=$ worst pain).

All RSB procedures were performed by an experienced surgeon in the operating room before wound closure. The study protocol with the exclusion criteria and informed consent were fully described in our earlier original works (6-9).

EDTA-blood samples were taken at the prespecified timepoints and centrifuged at $1000 \mathrm{~g}$ for $15 \mathrm{~min}$. Plasma was separated and stored frozen at $-70^{\circ} \mathrm{C}$ until analyzed. The plasma SOD1 assays were performed using sandwich-type ELISA methods from BioVendor $\mathrm{Cu} / \mathrm{Zn}$ SOD ELISA Kit (Brno, Czech Republic, www.biovendor.com). The plasma catalase assays were performed using enzyme-linked immunosorbent assay ELISA Kit (CloudClone Corporation, Katy, TX, USA). The plasma NT assays were performed using competitive enzyme immunoassay OxiSelect ${ }^{\mathrm{TM}}$ Nitrotyrosine ELISA Kit (STA-305, Cell Biolabs Inc, San Diego, CA, USA). The manufacturer's intra-assay and the inter-assay Cvs were $5.1 \%$ and $5.8 \%$, respectively.
The data were entered and analysed with a statistical software program (IBM SPSS Statistics 24.0; IBM, Armonk, NY, USA). Differences in baseline characteristics between groups were tested by Fisher's exact test and in the case of continuous data, the analysis was performed by Kruskall-Wallis $t$-test. Group differences at three time points were tested by the Wilcoxon signed rank test and the Kruskall-Wallis $t$-test. The plasma SOD1, CAT and NT concentrations and the differences in the patients with benign diseases and cancer were tested by the Mann-Whitney $U$-test. The results of the laboratory measurements are presented as median concentrations with interquartile range as distributions were rightskewed. A two-sided $p$-value of less than 0.05 was considered statistically significant. Pearson's method was used to test for correlation of NT values versus individual patient satisfaction following surgery $\left(\mathrm{SFS}_{24}\right)$, and pain at rest and under pressure $\left(\mathrm{NRS}_{\mathrm{r}}\right.$ and $\left.\mathrm{NRS}_{\mathrm{p}}\right)$.

\section{Results}

The clinical data of patients with benign disease and cancer were quite similar in the four study groups, although patients' weight and body mass index (BMI) were significantly higher in the single dose and control group than in the two other study groups $(p=0.007$ and $p=0.03$, respectively, Table I). The study group consisted of 15 patients with benign disease, nine patients with gastrointestinal cancer, 17 patients with gynaecological cancer and three patients with other malignancy (Table I). The RSB analgesia enhanced significantly the median $\mathrm{SFS}_{24}$ scores in the following order; control, single dose, repeated dose and continuous dose group $(8 ; 9 ; 9.5$ and 10 , respectively, $p=0.001$, Table I).

There were no statistically significant differences detected in the SOD1, CAT and NT values between the control group patients and the three active groups preoperatively and following surgery. Although, the patients in the single dose group had a trend for higher median NT levels preoperatively and immediately after operation (POP1) compared to the control group, repeated dose and continuous dose groups (median plasma concentrations $\mathrm{pg} / \mathrm{ml}, 7.15 / 7.74$ versus 5.47/4.03 and 5.28/5.54 and 6.25/5.96, respectively, Table II).

The postoperative alteration of plasma SOD1, CAT and NT marker concentrations in the groups combined are shown in Table III. There were statistically significant increases in the median plasma concentrations of SOD1 and CAT preoperatively (PRE) and immediately after operation (POP1) ( $p=0.007$ and $p<0.001$, respectively, Table III). Then the median plasma concentrations of SOD1 and CAT markers decreased $24 \mathrm{~h}$ postoperatively (POP2) and the decrease between the POP1 and POP2 values in the SOD1 and CAT markers were statistically highly significant (43\% decrease, $p<0.001$ and $24 \%$ decrease, $p=0.001$, respectively, Table III). There was no significant alteration in the median (interquartile range) plasma concentrations of NT preoperatively (PRE) and 


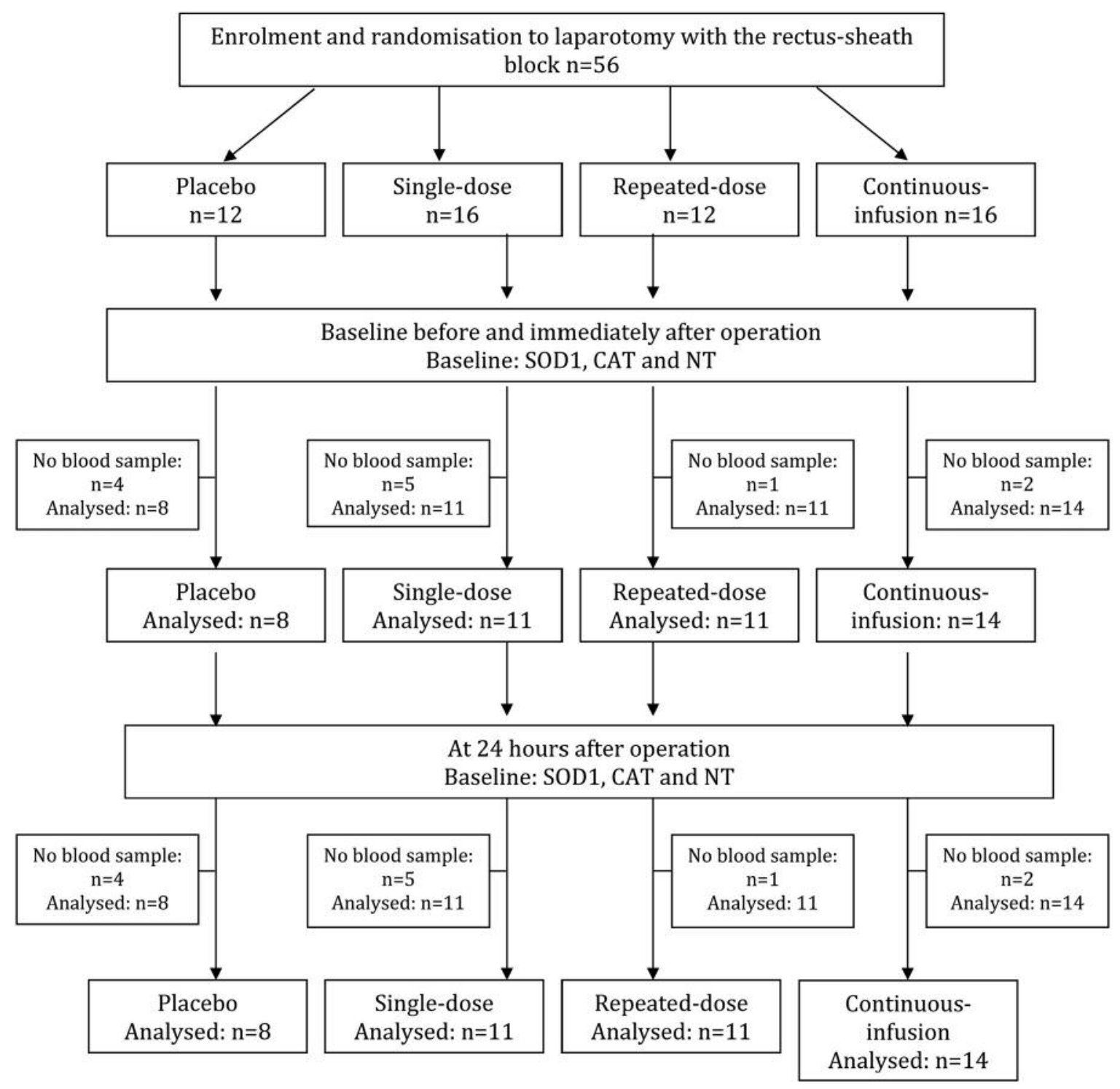

Figure 1. Design of the study as a flowchart.

immediately after operation (POP1) $(p=0.361$, Table III). However, the median plasma concentrations of NT decreased $24 \mathrm{~h}$ postoperatively (POP2) and the decrease between the POP1 and POP2 values in the NT were statistically significant ( $17 \%$ decrease, $p=0.041$, Table III).

The median (interquartile range) plasma concentrations of SOD1 and CAT did not differ significantly between the benign and cancer patients, preoperatively or after operation (Table IV). Patients in the cancer group had a trend for lower median NT levels compared to the benign patients (Table IV). However, there was a statistically significant difference in median NT concentrations between benign and cancer patients immediately following surgery (POP1) $(p=0.008$, Table IV).

The median (interquartile range) plasma concentrations of SOD1 and CAT did not correlate significantly to NT concentrations in benign and cancer patients (data not shown). Figure 2 shows scatter plots of the plasma NT concentrations versus patient satisfaction assessed using a $\mathrm{SFS}_{24}$ at $24 \mathrm{~h}$ following surgery in benign and cancer patients $(\mathrm{r}=-0.284, p=0.028)$. There was a statistically significant negative correlation in median plasma concentrations of NT versus an increase in patient satisfaction between PRE and POP2 ( $\mathrm{r}=-0.454, p=0.010)$ (data not shown). 
Table I. The midline laparotomy patients' clinical data in the four study groups. Data are mean (standard deviation) or number of cases.

\begin{tabular}{|c|c|c|c|c|c|}
\hline \multirow[b]{2}{*}{ Variable } & \multicolumn{5}{|c|}{ RSB group } \\
\hline & $\begin{array}{c}\text { Control } \\
n=8\end{array}$ & $\begin{array}{c}\text { Single } \\
\mathrm{n}=11\end{array}$ & $\begin{array}{c}\text { Repeated } \\
\mathrm{n}=11\end{array}$ & $\begin{array}{c}\text { Continuous } \\
\mathrm{n}=14\end{array}$ & $p$-Value \\
\hline Age, years & $62.6(14.3)$ & $60.8(12.6)$ & $63.3(10.8)$ & $58.0(10.1)$ & 0.74 \\
\hline Gender male/female & $4 / 4$ & $4 / 7$ & $2 / 9$ & $2 / 12$ & 0.22 \\
\hline Height, $\mathrm{cm}$ & $166.6(8.6)$ & $168.4(7.9)$ & $165.7(7.2)$ & $164.3(6.6)$ & 0.62 \\
\hline Weight, $\mathrm{kg}$ & $78.6(11.8)$ & $83.7(12.8)$ & $67.8(13.7)$ & $68.8(10.6)$ & 0.007 \\
\hline $\mathrm{BMI}, \mathrm{kg} / \mathrm{m} 2$ & $28.3(3.8)$ & $29.6(4.4)$ & $24.6(4.3)$ & $25.7(4.9)$ & 0.03 \\
\hline Time in the operative room, min & $229.4(113.4)$ & $274.9(148.4)$ & $235.7(112.0)$ & $279.7(178.5)$ & 0.85 \\
\hline Operative time, $\min$ & $209.6(141.2)$ & $221.8(156.4)$ & $154.4(95.0)$ & $253.3(168.9)$ & 0.55 \\
\hline Perioperative-bleed, $\mathrm{ml}$ & $696(741)$ & $822(906)$ & $697(967)$ & $1340(928)$ & 0.31 \\
\hline ASA $1 / 2 / 3 / 4$ & $0 / 6 / 2 / 0$ & $0 / 7 / 3 / 1$ & $0 / 5 / 6 / 0$ & $2 / 7 / 5 / 0$ & 0.43 \\
\hline $\mathrm{NRS}_{\mathrm{r}}$ & $2(1-6)$ & $2(0-4)$ & $2(0-3)$ & $1(0-4)$ & 0.41 \\
\hline $\mathrm{NRS}_{\mathrm{p}}$ & $5(3-9)$ & $5(1-9)$ & $3.5(1-7)$ & $4(0-7)$ & 0.42 \\
\hline $\mathrm{SFS}_{24}$ & $8(7-8)$ & $9(8-10)$ & $9.5(8-10)$ & $10(8.3-10)$ & 0.001 \\
\hline Length of the skin incision(s), mm & $27.2(6.6)$ & $24.4(7.8)$ & $24.2(7.9)$ & $29.7(7.3)$ & 0.31 \\
\hline Type of disease & & & & & 0.32 \\
\hline Benign $(n=15)$ & 1 & 3 & 5 & 6 & \\
\hline GI cancer $(n=9)$ & 3 & 2 & 2 & 2 & \\
\hline Gyn cancer $(n=17)$ & 3 & 4 & 4 & 6 & \\
\hline Other cancer $(n=3)$ & 1 & 2 & 0 & 0 & \\
\hline
\end{tabular}

GI: Gastrointestinal tract; Gyn: gynaecological; BMI: body mass index; ASA: American Society of Anesthesiologists physical status score; NRS: 11-point numeric rating scale $24 \mathrm{~h}$ after surgery; $\mathrm{NRS}_{\mathrm{r}}$ : NRS at rest; $\mathrm{NRS}_{\mathrm{p}}$ : NRS under $2 \mathrm{~kg}$ pressure to the wound area; $\mathrm{SFS}_{24}$ : satisfaction following surgery; RSB: rectal sheath block.

Table II. The plasma superoxide dismutase 1 (SOD1), catalase (CAT) and nitrotyrosine (NT) concentrations in the four study groups. Plasma concentrations were measured before (PRE), immediately after (POP1) and 24 h after (POP2) surgery. Median (interquartile range) concentrations are shown.

\begin{tabular}{|c|c|c|c|c|c|}
\hline \multirow[b]{2}{*}{ Marker } & \multicolumn{5}{|c|}{ RSB group } \\
\hline & Control & Single & Repeated & Continuous & $p$-Value* \\
\hline \multicolumn{6}{|c|}{ SOD1 (pg/ml) } \\
\hline PRE & $150(101-288)$ & $146(96-287)$ & $171(113-216)$ & 168 (128-199) & 0.922 \\
\hline POP1 & $281(144-522)$ & $160(101-305)$ & $184(123-275)$ & $243(151-292)$ & 0.392 \\
\hline POP2 & $119(93-202)$ & $87(59-105)$ & $116(88-171)$ & $167(94-217)$ & 0.138 \\
\hline \multicolumn{6}{|c|}{ CAT $(\mathrm{pg} / \mathrm{ml})$} \\
\hline PRE & $0.95(0.67-2.36)$ & $0.74(0.45-1.58)$ & $0.61(0.41-1.09)$ & $0.61(0.43-1.17)$ & 0.407 \\
\hline POP1 & $1.36(0.89-3.71)$ & $1.89(0.78-2.93)$ & $1.53(0.78-2.17)$ & $1.39(0.84-2.03)$ & 0.907 \\
\hline POP2 & $1.17(0.58-1.65)$ & $0.86(0.60-1.73)$ & $1.15(0.77-1.50)$ & $1.06(0.83-1.92)$ & 0.891 \\
\hline \multicolumn{6}{|c|}{$\mathrm{NT}(\mathrm{pg} / \mathrm{ml})$} \\
\hline PRE & $5.47(3.03-6.01)$ & $7.15(5.84-8.81)$ & $5.28(4.00-8.74)$ & $6.25(5.09-8.78)$ & 0.128 \\
\hline POP1 & $4.03(2.98-5.93)$ & $7.74(5.96-9.16)$ & $5.54(3.21-7.21)$ & $5.96(4.30-8.43)$ & 0.127 \\
\hline POP2 & $4.39(3.24-7.55)$ & $4.58(3.41-5.87)$ & $4.70(4.15-5.51)$ & $6.37(4.55-7.31)$ & 0.277 \\
\hline
\end{tabular}

*The Kruskall-Wallis test.

\section{Discussion}

The NT plasma concentrations versus patient satisfaction $24 \mathrm{~h}$ following surgery $\left(\mathrm{SFS}_{24}\right)$ in midline laparotomy patients with RSB analgesia is unknown. The original study hypothesis was that RSB analgesia could lower postoperative pain following surgery and enhance satisfaction in benign and cancer patients. Interestingly, in the present study, patients in the continuous infusion group had a trend for lower pain score at rest $\left(\mathrm{NRS}_{\mathrm{r}}\right)$ compared to other groups. However, no statistically significant differences were detected in the pain scores; NRS pain at rest and under pressure $\left(\mathrm{NRS}_{\mathrm{r}}\right.$ and $\left.\mathrm{NRS}_{\mathrm{p}}\right)$ 
Table III. The postoperative alteration of plasma superoxide dismutase 1 (SOD1), catalase (CAT) and nitrotyrosine (NT) concentrations in all groups combined (all patients). Plasma concentrations were measured before (PRE), immediately after (POP1) and 24 h after (POP2) surgery. Median (interquartile range) concentrations are shown.

\begin{tabular}{lccr}
\hline Marker & All patients & Alteration & $p$-Value* \\
\hline SOD1 $(\mathrm{pg} / \mathrm{ml})$ & & & \\
$\quad$ PRE & $164.0(114-213)$ & & \\
POP1 & $185.0(137-301)$ & PRE $v s$. POP1 & 0.007 \\
POP2 & $105.0(86-176)$ & POP1 vs. POP2 & $<0.001$ \\
CAT $(\mathrm{pg} / \mathrm{ml})$ & & & \\
PRE & $0.66(0.48-1.17)$ & & \\
POP1 & $1.39(0.84-2.43)$ & PRE $v s$. POP1 & $<0.001$ \\
POP2 & $1.03(0.70-1.57)$ & POP1 vs. POP2 & 0.001 \\
NT (pg/ml) & & & \\
PRE & $6.02(4.78-8.16)$ & & \\
POP1 & $5.96(4.14-8.03)$ & PRE $v s$. POP1 & 0.361 \\
POP2 & $4.96(4.12-6.53)$ & POP1 vs. POP2 & 0.041 \\
\hline
\end{tabular}

*Wilcoxon signed-rank test.

Table IV. The plasma superoxide dismutase 1 (SOD1), catalase (CAT) and nitrotyrosine (NT) concentrations in patients with benign diseases $(n=15)$ and those with cancer $(n=29)$. Plasma concentrations were measured before (PRE), immediately after (POPl) and $24 \mathrm{~h}$ after $(P O P 2)$ surgery. Median (interquartile range) concentrations are shown.

\begin{tabular}{lccc}
\hline Marker & Benign & Cancer & $p$-Value* \\
\hline SOD1 (pg/ml) & & & \\
PRE & $167(132-216)$ & $160(107-211)$ & 0.647 \\
POP1 & $182(135-286)$ & $202(136-338)$ & 0.989 \\
POP2 & $105(76-171)$ & $112(86-196)$ & 0.610 \\
CAT (pg/ml) & & & \\
PRE & $0.62(0.41-1.17)$ & $0.73(0.48-1.30)$ & 0.407 \\
POP1 & $1.09(0.78-2.50)$ & $1.46(1.13-2.36)$ & 0.304 \\
POP2 & $0.83(0.75-1.65)$ & $1.12(0.68-1.50)$ & 0.593 \\
NT (pg/ml) & & & \\
PRE & $6.83(5.05-8.32)$ & $5.67(4.10-8.10)$ & 0.250 \\
POP1 & $7.58(5.96-9.71)$ & $5.28(3.41-6.44)$ & 0.008 \\
POP2 & $5.75(4.35-6.73)$ & $4.69(4.09-6.27)$ & 0.308 \\
\hline
\end{tabular}

*Mann-Whitney $U$-test.

between the control group and three RSB study groups showing that our main hypothesis is not fully realized. In contrary, statistically significant differences were detected in the patient satisfaction $24 \mathrm{~h}$ following surgery $\left(\mathrm{SFS}_{24}\right)$ between the control group and three RSB study groups supporting our main hypothesis. Our results are in line with an earlier study assessing patient satisfaction after various surgical procedures, which indicated that even though $62 \%$ of patients experienced severe postoperative pain, $87 \%$ of them were satisfied with pain treatment (10). Owen et al. 1990 (11) and Svensson et al. 2001 (12) have also reported this finding of patient satisfaction.

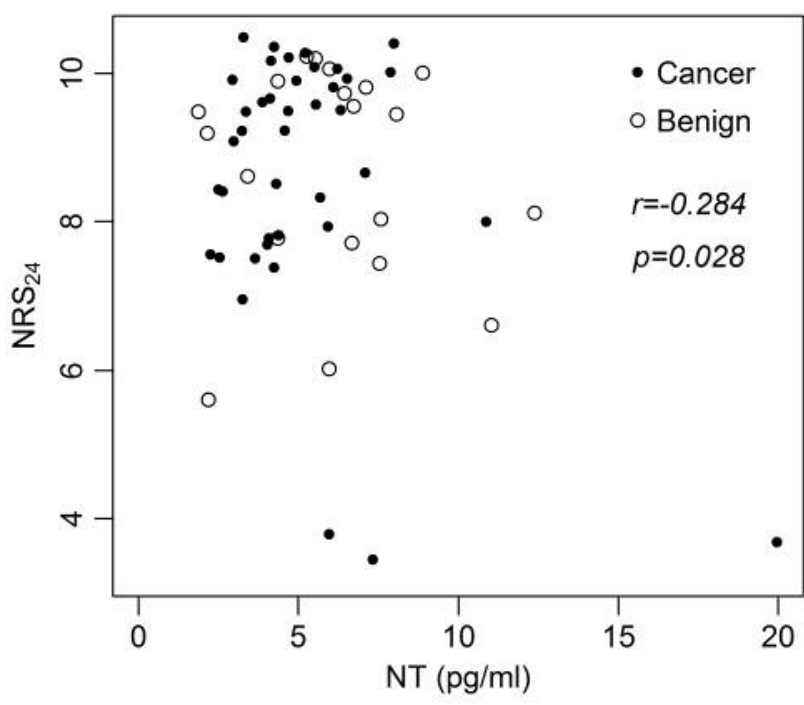

Figure 2. Scatter plots of the plasma nitrotyrosine (NT) concentrations versus patient satisfaction assessed using a 11-point rating scale $\left(\mathrm{SFS}_{24} ; 0=\right.$ fully unsatisfied; 10=fully satisfied) at $24 \mathrm{~h}$ following surgery in benign and cancer patients $(r=-0.284, p=0.028)$.

NT is a fingerprint of peroxynitrite-mediated nitration, and the presence of NT has been identified in $H$. pylori gastritis (13), and several human cancers as lung carcinoma (14), cholangiocarcinoma (15), pancreatic carcinoma (16), esophageal carcinoma (17) and melanoma (18). It is very difficult to directly measure the peroxynitrite concentration in plasma, therefore NT is an available biomarker for indirect detection of peroxynitrite concentration. In earlier studies NT was detected in plasma, urine and bronco-alveolar fluid. In the present study the patients in the cancer group had a trend for lower median NT levels compared to the benign patients and there was statistically significant difference in median NT concentrations between benign and cancer patients immediately following surgery (POP1). In addition, patients in the single dose group had a trend for higher plasma NT concentrations preoperatively (PRE) and immediately following surgery (POP1) compared to other study groups. We have reported earlier that midline laparotomy with RSB significantly enhances the concentration of the oxidative stress markers SOD1 and CAT immediately after operation, but both marker concentrations drop back $24 \mathrm{~h}$ following surgery $(2,9)$. RSB analgesia could be effective in midline laparotomy, where RSB is suggested as a part of a multimodal postoperative analgesia (19).

Also, we recently reported that cholecystectomy patients with enhanced concentrations of SOD1 and CAT appeared to have significantly lower analgesia requirement during the first $24 \mathrm{~h}$ postoperatively $(3,20)$. In this study, the most important finding is the significant correlation between plasma NT concentrations and $\mathrm{SFS}_{24}$ following surgery in benign and 
cancer patients. In contrary, plasma NT concentrations decreased significantly $24 \mathrm{~h}$ following surgery (POP2).

The blinding of the different RSB analgesia groups was challenging. We decided not to use an invasive placebo, which meant that the control group was without RSB catheterization, however the patients in the control group had no local anaesthetic used and they were blinded using similar wound dressing to that of the patients in the active study groups. The blinding between the control group and RSB study groups could have been done with normal saline infiltrations, however invasive placebo with normal saline could have affected ROS/NRS mediators as well as pain scores.

In conclusion, it is important to develop ROS/RNS biomarkers in combination with clinical variables used as predictors of patient satisfaction and pain following surgery $(21,22)$. In earlier studies, the most promising clinical role of nitric oxide and NT as clinical biomarkers has been in neurodegenerative (23) and neuropsychiatric disorders (24), brain aging (23) and the understanding of the mechanisms of inflammation-associated colorectal cancer (25). The most important finding of the present study is that NRS analgesia could significantly enhance the patient satisfaction following midline laparotomy. In addition, it seems that plasma NT concentration is significantly correlated with patient satisfaction following surgery in benign disease and cancer.

\section{Conflicts of Interest}

The Authors report no conflicts of interest or financial ties to disclose. The authors alone are responsible for the content and writing of this article.

\section{Authors' Contributions}

All Authors have met all of the following four criteria: 1. Substantial contributions to the conception or design of the work or the acquisition, analysis, or interpretation of data for the work, 2. Drafting the work or revising it critically for important intellectual content, 3. Final approval of the version to be published, 4. Agreement to be accountable for all aspects of the work in ensuring that questions related to the accuracy or integrity of any part of the work are appropriately investigated and resolved.

\section{Acknowledgements}

The study was funded by the Heikki, Aino and Aarne Korhonen foundation and the EVO-funding of the Kuopio University Hospital, Finland.

\section{References}

1 Sundbom $M$ and Hedberg $J$ : Use of Laparoscopy in Gastrointestinal Surgery in Sweden 1998-2014: A Nationwide Study. Scand J Surg 106: 34-39, 2017. PMID: 26929297, DOI: $10.1177 / 1457496916630645$
2 Saimanen I, Kärkkäinen J, Selander T, Purdy M, Kokki M, Kokki $\mathrm{H}$ and Eskelinen M: Plasma catalase in relation to pain following midline laparotomy: a prospective study of patients with benign diseases and patients with cancer. Anticancer Res 38: 6479-6484, 2018. PMID: 30396975, DOI: 10.21873/anticanres.13011

3 Saimanen I, Kuosmanen V, Kärkkäinen J, Selander T, Aspinen S, Holopainen A, Rantanen T and Eskelinen M: Cholecystectomy patients with high plasma level of catalase have significantly lower analgesia requirement: a prospective study of two different cholecystectomy techniques with special reference to patients with cancer. Anticancer Res 38: 5417-5422, 2018. PMID: 30194197 , DOI: $10.21873 /$ anticanres.12872

4 Arsalani-Zadeh R, Ullah S, Khan S and MacFie J: Oxidative stress in laparoscopic versus open abdominal surgery: A systematic review. J Surg Res 169: 59-68, 2011. PMID: 21492871, DOI: 10.1016/j.jss.2011.01.038

5 Yiannakopoulou ECh, Nikiteas N, Perrea D and Tsigris C: Effect of laparoscopic surgery on oxidative stress response: systematic review. Surg Laparosc Endosc Percutan Tech 23: 101-108, 2013. PMID: 23579502, DOI: 10.1097/SLE.0b013e3182827b33

6 Purdy M, Kokki M, Anttila M, Aspinen S, Juvonen P, Selander T, Kokki H, Pulkki K and Eskelinen M: Does the post-surgery placement of rectus sheath block analgesia alter the oxidative stress biomarker 8-OHdG concentrations: A randomised trial of patients with cancer and benign disease. Cancer Genomics Proteomics 13: 239-244, 2016. PMID: 27107066.

7 Purdy M, Kokki M, Anttila M, Aspinen S, Juvonen P, Korhonen R, Selander T, Kokki H and Eskelinen M: Does the rectus sheath block analgesia reduce the inflammatory response biomarker IL1ra, IL-6, IL-8, IL-10 and IL-1 $\beta$ concentrations following surgery? A randomised clinical trial of patients with cancer and benign disease. Anticancer Res 36: 3005-3011, 2016. PMID: 27272818.

8 Purdy M, Kärkkäinen J, Kokki M, Anttila M, Aspinen S, Juvonen P, Kokki H, Pulkki K, Rantanen T and Eskelinen M: Does rectus sheath block analgesia alter levels of the oxidative stress biomarker glutathione peroxidase: a randomised trial of patients with cancer and benign disease. Anticancer Res 37: 897-902, 2017. PMID: 28179349, DOI: 10.21873/anticanres.11396

9 Kärkkäinen J, Selander T, Purdy M, Juvonen P and Eskelinen M: Patients with increased levels of the oxidative stress biomarker SOD1 appear to have dimished postoperative pain after midline laparotomy: A randomized trial with special reference to postoperative pain score (NRS). Anticancer Res 38: 1003-1008, 2018. PMID: 29374733, DOI: 10.21873/ anticanres.12315

10 Sauaia A, Min SJ, Leber C, Erbacher K, Abrams F and Fink R: Postoperative pain management in elderly patients: correlation between adherence to treatment guidelines and patient satisfaction. J Am Geriatr Soc 53: 274-282, 2005. PMID: 15673352.

11 Owen H1, McMillan V and Rogowski D: Postoperative pain therapy: a survey of patients' expectations and their experiences. Pain 41: 303-307, 1990. PMID: 1697058.

12 Svensson I, Sjöström B and Haljamäe H: Influence of expectations and actual pain experiences on satisfaction with postoperative pain management. Eur J Pain 5: 125-133, 2001. PMID: 11465978.

13 Sasaki T, Kuniyasu H, Luo Y, Kitayoshi M, Tanabe E, Kato D, Shinya S, Fujii K, Ohmori H and Yamashita Y: Increased phosphorylation of AKT in high risk gastric mucosa. Anticancer Res 33: 3295-3300, 2013. PMID: 23898095. 
14 Luanpitpong $\mathrm{S}$ and Chanvorachote $\mathrm{P}$ : Nitric oxide and aggressive behavior of lung cancer cells. Anticancer Res 35: 4585-4592, 2015. PMID: 26254346.

15 Jaiswal M, LaRusso NF, Burgart LJ and Gores GJ: Inflammatory cytokines induce DNA damage and inhibit DNA repair in cholangiocarcinoma cells by a nitric oxide-dependent mechanism. Cancer Res 60: 184-190, 2000. PMID: 10646872.

16 Vickers SM, MacMillan-Crow LA, Green M, Ellis C and Thompson JA: Association of increased immunostaining for inducible nitric oxide synthase and nitrotyrosine with fibroblast growth factor transformation in pancreatic cancer. Arch Surg 134: 245-251, 1999. PMID: 10088562.

17 Kato H, Miyazaki T, Yoshikawa M, Nakajima M, Fukai Y, Tajima K, Masuda N, Tsutsumi S, Tsukada K, Nakajima T and Kuwano $\mathrm{H}$ : Nitrotyrosine in esophageal squamous cell carcinoma and relevance to p53 expression. Cancer Lett 153: 121-127, 2000. PMID: 10779640.

18 Hintsala HR, Jokinen E, Haapasaari KM, Moza M, Ristimäki A, Soini Y, Koivunen J and Karihtala P: Nrf2/Keap1 Pathway and expression of oxidative stress lesions 8-hydroxy-2'deoxyguanosine and nitrotyrosine in melanoma. Anticancer Res 36: 1497-1506, 2016. PMID: 27069125.

19 Purdy M, Kinnunen M, Kokki M, Anttila M, Eskelinen M, Hautajärvi $\mathrm{H}$, Lehtonen $\mathrm{M}$ and Kokki $\mathrm{H}$ : A prospective, randomized, open label, controlled study investigating the efficiency and safety of 3 different methods of rectus sheath block analgesia following midline laparotomy. Medicine 97: e9968, 2018. PMID: 29443788, DOI: 10.1097/MD.0000000000009968

20 Kärkkäinen J, Saimanen I, Selander T, Aspinen S, Harju J, Juvonen $\mathrm{P}$ and Eskelinen M: Gallstone patients with enhanced oxidative stress biomarker SOD1 plasma levels have significantly lower number of postoperative analgesic oxycodone doses: A prospective study with special reference to cancer patients. Anticancer Res 38: 3573-3578, 2018. PMID: 29848712, DOI: 10.21873/anticanres. 12630
21 Seyidova D, Aliyev A, Rzayev N, Obrenovich M, Lamb BT, Smith MA, de la Torre JC, Perry G and Aliev G: The role of nitric oxide in the pathogenesis of brain lesions during the development of Alzheimer's disease. In Vivo 18: 325-333, 2004. PMID: 15341188.

22 Takumida $\mathrm{M}$ and Anniko M: Functional significance of nitric oxide in the inner ear. In Vivo 18: 345-350, 2004. PMID: 15341190.

23 Calabrese V, Boyd-Kimball D, Scapagnini G and Butterfield DA: Nitric oxide and cellular stress response in brain aging and neurodegenerative disorders: the role of vitagenes. In Vivo 18: 245-267, 2004. PMID: 15341181.

24 Akyol O, Zoroglu SS, Armutcu F, Sahin S and Gurel A: Nitric oxide as a physiopathological factor in neuropsychiatric disorders. In Vivo 18: 377-390. PMID: 15341194.

25 Mladenova D and Kohonen-Corish MR: Review: Mouse models of inflammatory bowel disease - insights into the mechanisms of inflammation-associated colorectal cancer. In Vivo 26: 627646, 2012. PMID: 22773577.
Received February 4, 2019

Revised February 18, 2019

Accepted February 19, 2019 